\title{
An Integrated Review of Developmental Outcomes and Late-Preterm Birth
}

Haifa A. Samra, Jacqueline M. McGrath, and Michelle Wehbe

Correspondence

Haifa (Abou) Samra, PhD,

RN-NIC, South Dakota State

University, College of

Nursing, Box 2275, SNF

209, Brookings, SD 57007.

haifa.samra@sdstate.edu

Keywords

development

growth

late-preterm

near-term

function

psychosocial

learning

cognitive

long-term outcomes

\section{ABSTRACT}

Objective: To evaluate existing evidence on long-term developmental outcomes of late-preterm infants (LPI; infants born 34-36 6/7 weeks gestation).

Data Sources: Computerized bibliographic databases and hand search for English language articles published between January 1995 and November 2010 yielded 817 articles.

Study Selection: Twelve studies (10 cohort and two cross-sectional) were identified that defined late-preterm (LP) birth as 34 to 36 6/7 weeks gestation and addressed growth and neurodevelopmental outcomes in LPI.

Data Extraction: Using a modified Downs and Black scale for assessing the quality of experimental and observational studies, two reviewers who were blind to each other's ratings assessed study quality. Ratings ranged from 12.5 to 14 with moderate to very good interrater agreement. Kappa $(\kappa)$ values were 0.83 (reporting), 0.63 (external validity), 0.73 (internal validity), and 0.83 (design) for the four subscales and 0.56 for the whole scale, with no major systematic disagreements between reviewers.

Data Synthesis: Studies were divided into five categories to include the following developmental outcomes: neurodevelopment, behavioral, cognitive, growth, and function. Using the Meta-analysis of Observational Studies in Epidemiology (MOOSE) guidelines, synthesis of the findings is provided as an integrative review.

Conclusion: Significant variations in study populations, methodology, and definition of LP exist. Due to paucity and heterogeneity of the existing data especially in infants born 34 to 36 6/7 weeks, there is no clear characterization of the long-term risks, and future research is needed.

JOGNN, 40, 399-411; 2011. DOI: 10.1111/j.1552-6909.2011.01270.x
Haifa A. Samra, PhD, RN-NIC, is an assistant professor in the College of Nursing, South Dakota State University, Brookings, SD.

Jacqueline M. McGrath, $\mathrm{PhD}$, RN, FNAP, FAAN, is an associate professor, in the School of Nursing, Virginia Commonwealth University, Richmond, VA.

Michelle Wehbe is a student at the University of Michigan, Ann Arbor, MI.

The authors report no conflict of interest or relevant financial relationships.

AWHONN 1 )

http://jognn.awhonn.org $A_{36,6 / 7 \text { weeks gestation) birth declined from }}^{\text {Ithough late preterm (LP: born between } 34 \text { and }}$ $12.8 \%$ to $12.3 \%$ over the past 2 years, incidence and subsequent consequences remain of great concern (Martin, Osterman, \& Sulton, 2010). Even though the risk of suboptimal outcomes decreases as gestation increases, any birth that takes place at fewer than 37 weeks gestation or fewer than 259 days from the first day of the mother's last menstrual period should be considered at risk (Engle, Tomashek, Wallman, \& American Academy of Pediatrics Committee on Fetus and Newborn, 2008). Accounting for $70 \%$ to $75 \%$ of all singleton preterm births and $9 \%$ of all births in the United States, late preterm infants (LPIs) are cared for either as small full-term infants (FTIs) in the newborn nursery or as infants on the fringes of the preterm population with little regard for their unique needs in the neonatal intensive care environment. In the past, these infants were believed to have no higher risks for medical and developmental problems than FTIs.
However, more recent data show that they do have metabolic, neurological, and physiological immaturities that could put them at increased risk for morbidity and mortality (Bastek et al., 2008; Engle et al.; Tomashek, Shapiro-Mendoza, Davidoff, \& Petrini, 2007; Tonse, Higgins, Stark, \& Leveno, 2006; Wang, Dorer, Fleming, \& Catlin, 2004). Late-preterm infants have been found to be at a higher risk for readmission; reasons include poor feeding, temperature instability, dehydration, jaundice, and sepsis. Increased risk for school underachievement, learning difficulties, and social and behavioral problems is also reported in children born late preterm (Gurka, LoCasale-Crouch, \& Blackman, 2010; Morse, Zheng, Tang, \& Roth, 2009; Moster, Terje Lie, \& Markestad, 2008).

During the 34 to 36 6/7 week period of gestation, the human brain (including its neural structures and pathways) experiences a critical period of rapid growth. Thirty-five percent of the total fetal 


\section{There is a great need to better characterize the risks and to evaluate existing evidence on suboptimal growth and developmental outcomes in late-preterm infants.}

brain and $47 \%$ of the cortical volume is acquired during the last 6 weeks of gestation. The myelinated white matter undergoes a fivefold increase between 34 and 41 weeks gestation, and the grey matter volume increases at a rate of $1.4 \%$ or $15 \mathrm{ml} /$ week (Kinney, 2006). When an infant is born prior to 37 weeks gestation, accelerated brain growth occurs postnatally and without utero protection. The experiences are different, and thus the neuro networks that form are different. Several physiological, psychosocial, and environmental risk factors such as disease states, medical treatments, care environments, and caregiver characteristics and their interactions can impede healthy transition from utero, compound existing biological vulnerabilities, and therefore, adversely influence the progression of growth and development (Melnyk, Feinstein, \& Fairbanks, 2006; White-Traut \& Norr, 2009). As increasing attention is currently being directed toward improving outcomes and increasing awareness about the risks that are associated with LP birth, there remains a great need to better characterize the risks and to evaluate existing evidence on suboptimal growth and developmental outcomes.

In 2005 a workshop sponsored by the National Institute of Child Health and Human Development of the National Institute of Health to address the issues related to late-preterm birth provided a comprehensive review on short-term outcomes, made evidence-based recommendations for late-preterm infant care, and identified future research areas (Tonse et al., 2006). However, the workshop indicated that data on long-term neurodevelopmental outcomes in LPIs are lacking with a paucity of data on behavioral problems, learning disabilities, and neurological abnormalities. The purpose of this review is to examine how this gap has been addressed in the more recent literature and to evaluate the quality of existing evidence. Practice implications and research recommendations are also included.

\section{Objective}

Following a modified MOOSE guidelines for reporting Meta-analyses Of Observational Studies in Epidemiology when applicable (Stroup et al.,
2000), an analytical framework was developed to answer three key questions:

- Is there sufficient evidence to support a relationship between suboptimal growth and developmental outcomes and history of latepreterm birth?

- What is the quality of the evidence supporting such relationship?

- What types of growth and developmental problems are observed in the late-preterm infants and what are the gaps in existing knowledge?

\section{Data Sources and Extraction and Study Selection}

\section{Data Sources and Extraction}

In collaboration with a librarian, the authors conducted a search of MEDLINE, the Cochrane library, NLM gateway, Cumulative Index for Nursing and Allied Health Literature (CINAHL), Nursing Consult, Biological Abstracts Previews (BIOIS), Health Source: Nursing/Academic Edition, and HealthStar for English language articles published between January 1995 and January 2011. The search also included cross-references and references lists, abstracts, conference and symposia proceedings, and dissertations. The last search run was on October 20, 2010. Electronic database searches were based on the following MeSH search terms:

("Premature Birth"[Mesh] OR "Infant, Premature" [Mesh] OR "Infant, Premature, Diseases"[Mesh]) AND ("Longitudinal Studies"[Mesh:NoExp] OR "Follow-Up Studies"[Mesh]) AND ("Late preterm" OR moderately [ti] OR "near term" OR (week* [ti] AND ("34" [ti] OR "35" [ti] OR "36" [ti] OR "37" [ti]))) Limits: Publication Date from 1995 to 2010. This search was also repeated with the ("Longitudinal Studies"[Mesh:NoExp] OR "Follow-Up Studies" [Mesh]) term left out. The search was also done using the search string (preterm OR premature OR prematurity OR infant OR infants OR infancy OR newborn OR newborns OR neonatal OR neonate OR neonates OR baby OR babies OR nicu) AND ("Late preterm" OR "moderate prematurity" OR "moderately preterm" OR "moderately premature" OR nearterm OR "near term" OR (ti week* AND (ti "34" OR ti "35" OR ti "36" OR ti "37”).

\section{Study Selection}

After potential references were identified through electronic and manual searches, references were retrieved and screened, and relevant articles were 
kept for further review and synthesis. Inclusion criteria for this integrative review were studies related to growth, neurodevelopment outcomes, learning and cognitive outcomes and school performance, social and behavioral outcomes, neuro-sensory outcomes, functional and motor outcomes in infants, children, and young adults with a history of late-preterm (sometimes referred to in the studies as near-term) birth (34-36 6/7 weeks gestation). Studies that used birth weight as a cutoff measure to define maturity level without reference to gestational age and studies that did not control for symmetrical and asymmetrical intrauterine growth retardation were excluded from the analysis. Controlled trials and studies examining outcomes in infants who received nitric oxide and other therapies and treatment modalities as well as follow-up studies on hyperbilirubinemia and preexisting disorders and disease-related outcomes that would possibly increase the likelihood of a compromised neurodevelopmental outcome were also excluded. Two reviewers assessed study eligibility through discussions until consensus about inclusion was achieved.

A total of 407 publications with no relevant systematic reviews were retrieved and screened. After screening was complete, 355 publications were excluded and those are described as expert opinion, recommendation statements, review articles, intervention studies, and mortality comparisons. Of the remaining 52 records, 40 did not meet the inclusion criteria. Those are described as studies that expanded the late-preterm definition to include infants born at 32 and 33 weeks gestation, studies that stratified comparison groups by disease outcomes, severity of condition at birth, delivery room interventions and APGAR scores, and birth weight with no mention of gestational age.

The search yielded 12 eligible studies that met the definition of 34 to 36 6/7 weeks gestation that was recommended by the 2005 workshop "Optimizing Care and Outcome of the Near-Term Pregnancy and Near-Term Newborn Infant" (Engle et al., 2008) and that addressed growth and neurodevelopmental outcomes in LPI. Ten were cohort (Baron, Erickson, Ahronovich, \& Coulehan, 2009; Eide, Oyen, Skjaerven, \& Bjerkedal, 2007; Gurka et al., 2010; Morse et al., 2009; Moster et al., 2008; Petrini et al., 2009; Romeo et al., 2007, 2009; Santos et al., 2009; Talge et al., 2010) and two were cross-sectional studies ([Abou] Samra, Binkley, Stevens, \& Specker, 2009; Barros, Mitsuhiro, Chalem, \& Laranjeira, 2010). Table 1 presents a summary of the 12 studies. To address the first research question: "Is there sufficient evidence to support the relationship between suboptimal growth and developmental outcomes and history of LP birth"? We found only a small number of studies examined growth and developmental outcomes in LPI in comparison with FTI. Current evidence is inadequate and is limited to only 12 observational studies. Future prospective studies on long-term growth and developmental outcomes in LPI are urgently needed.

\section{Assessment of Study Quality}

To answer the second question regarding study quality, a modified Downs and Black scale (Downs \& Black, 1998) was used to assess the quality of review studies that met the inclusion criteria. The Downs and Black scale has been designed to assess the quality of experimental and observational studies and provides an objective way to better examine the quality of the outcomes of these study designs and thus the reliability of the outcomes. The Agency for Healthcare Research and Quality rated the scale as one of high quality and credibility (West et al., 2002). The scale is reported to have a good test-retest $(r=.88)$ and interrater reliability $(r=.90)$. The authors modified the scale to fit the review topic (see Table 2). Studies were scored independently by two authors, and then discrepancies were discussed and resolved. Final scores are shown in Table 1. Interrater agreement statistic ( $\kappa)$ was used to evaluate the agreement between ratings for both authors prior to discussion of results. The strength of agreement ranged from moderate to very good. $\kappa$ values were 0.83 for reporting, 0.63 for external validity, 0.73 for internal validity, 0.83 for study design, and 0.56 for total score. There were no major systematic disagreements. Disagreements were mainly in the external and internal validity scores. Differences in external validity scores were related to how representative study samples were of the LP population. Differences in internal validity ratings were related to the impact of rate of attrition on the outcome and on whether blinding of individuals who performed the assessments was adequate and to the number and type of covariates that were considered to be of importance in confounding results as shown in Table 2.

\section{Data Synthesis}

\section{Summary of Evidence}

To address the third research question, the authors developed a framework of five developmental domains used to organize the review studies according to their outcome measures. Studies that examined motor development and neurological outcomes such as mental retardation, epilepsy, 


\section{Table 1: Summary of Published Studies and Their Quality Scores by \\ Developmental Domain}

\begin{tabular}{|c|c|c|c|c|}
\hline $\begin{array}{l}\text { Author/Design// } \\
\text { Location }\end{array}$ & Sample & Variables & Significant Findings & $\begin{array}{l}\text { Quality } \\
\text { Score }\end{array}$ \\
\hline \multicolumn{5}{|c|}{ Neuro-developmental and motor outcomes } \\
\hline $\begin{array}{l}\text { Petrini et al. (2009) } \\
\text { Retrospective cohort } \\
\text { study with } 5.5 \text { years } \\
\text { follow up } \\
\text { Location: United } \\
\text { States }\end{array}$ & $\begin{array}{l}\text { LP: } N=8,341 \text { (34-36 wks) } \\
\qquad F T: N=128,955 \text { (37-41 wks) } \\
\text { Age: } 5.5 \text { years, born } \\
\text { between Jan 1, } 2000 \text { and } \\
\text { June 30, 2004) } \\
\text { Follow-up period extended } \\
\text { through June 30, } 2005 . \\
\text { GA: Determined by } \\
\text { ultrasound scanning } \\
\text { between } 12 \text { and } 24 \text { wks }\end{array}$ & $\begin{array}{l}\text { Outcome measures: } \\
\text { Neurological diagnoses of } \\
\text { cerebral palsy (CP), seizure } \\
\text { disorder, and developmental } \\
\text { delays (DD) and mental } \\
\text { retardation (MR) }\end{array}$ & $\begin{array}{l}\text { LP children were three times } \\
\text { more likely to be diagnosed } \\
\text { with CP. } \\
\text { Adjusted hazard ratio } \\
(\mathrm{HR})=3.39(95 \% \mathrm{Cl}[2.54 \text {, } \\
4.52]) \text {. } \\
\text { Risk for DD/MR was marginally } \\
\text { higher than FT singleton } \\
\text { children. HR = } 1.25 \text { ( } 95 \% \mathrm{Cl} \\
[1.01,1.54]) \text {. }\end{array}$ & 15 \\
\hline $\begin{array}{l}\text { Romeo et al. (2009) } \\
\text { Retrospective and } \\
\text { part of a large } \\
\text { prospective cohort } \\
\text { follow-up study } \\
\text { (secondary analysis) } \\
\text { Location: Italy }\end{array}$ & 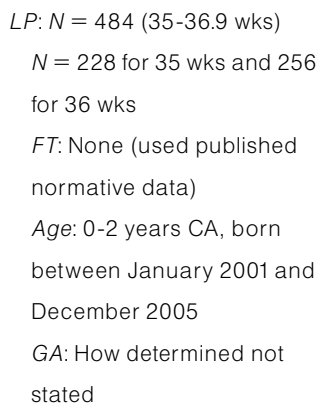 & $\begin{array}{l}\text { Outcome measure: Walking } \\
\text { age } \\
\text { Predictive variable: Forward } \\
\text { parachute reaction (FPR) } \\
\text { assessed at } 3.6,9 \text {, and } 12 \\
\text { months of corrected age. }\end{array}$ & $\begin{array}{l}\text { Patterns of FPR development } \\
\text { and independent walking } \\
\text { were similar in both in } 35 \text { and } \\
36 \text { wks and similar to } \\
\text { published normative data for } \\
\text { FTIs. }\end{array}$ & 14 \\
\hline $\begin{array}{l}\text { Moster et al. (2008) } \\
\text { Design } \\
\text { Prospective follow- } \\
\text { up analysis } \\
\text { Location: Norway }\end{array}$ & $\begin{array}{l}\text { LP: } N=32,945(34-36 \text { wks }) \\
\qquad F T: N=858,406 \\
(\geq 37 \text { wks) } \\
\text { Age: } 20-36 \text { years, born } \\
\text { between } 1967-1983 \\
\text { GA: Determined by last } \\
\text { menstrual period }\end{array}$ & $\begin{array}{l}\text { Outcome measures: } \\
\text { Prevalence of CP and MR, } \\
\text { educational attainment, } \\
\text { income, Social Security } \\
\text { benefits, disability pension, } \\
\text { establishment of family, } \\
\text { unemployment rates, and } \\
\text { criminal activity }\end{array}$ & $\begin{array}{l}\text { Risk of medical disabilities } \\
\text { including CP, MR, increased } \\
\text { as gestational age } \\
\text { decreased. } \\
\text { No direct comparisons } \\
\text { between LP and FT born } \\
\text { participants were made. }\end{array}$ & 14 \\
\hline $\begin{array}{l}\text { Romeo et al. (2007) } \\
\text { Retrospective } \\
\text { secondary analysis } \\
\text { of data from a larger } \\
\text { prospective cohort } \\
\text { follow-up study } \\
\text { Location: Italy }\end{array}$ & $\begin{array}{l}\text { LP: N }=448 \text { (35-36.9 wks) } \\
\text { FT: None (the Frequency } \\
\text { distribution of the } \\
\text { Hammersmith Infant } \\
\text { Neurological Examination } \\
\text { (HINE) scores was used for } \\
\text { comparison) } \\
\text { Age: } 6,9 \text {, and12 months CA, } \\
\text { born between January } \\
2000 \text { and December } 2004 \\
\text { GA: How determined not } \\
\text { stated }\end{array}$ & $\begin{array}{l}\text { Outcome measures: } \\
\text { Hammersmith Infant } \\
\text { Neurological Examination } \\
\text { (HINE) scores }\end{array}$ & $\begin{array}{l}\text { There was no difference in HINE } \\
\text { scores between } 35 \text { and } 36 \\
\text { wks infants } \\
\text { compared to full term infants } \\
\text { born at } 35 \text { and at } 36 \text { wks; } \\
\text { gestation showed } \\
\text { wider variability of scores } \\
\text { Lower scores in the subsections } \\
\text { of tone and reflexes at } 12 \\
\text { months corrected age } \\
\text { Similar scores for cranial } \\
\text { nerves, movements and } \\
\text { posture Lower mean and 10th } \\
\text { percentile HINE global scores }\end{array}$ & 12.5 \\
\hline \multicolumn{5}{|c|}{ Neuro-behavioral outcomes } \\
\hline $\begin{array}{c}\text { Barros et al. (2010) } \\
\text { Cross-sectional }\end{array}$ & $\begin{array}{l}\text { LP: } N=36(34-366 / 7 \mathrm{wks}) \\
F T: N=96(40-406 / 7 \mathrm{wks}) \\
\text { Age: } 24-72 \text { hours of life, }\end{array}$ & $\begin{array}{l}\text { Outcome variable(s): Neuro- } \\
\text { behavior using the Neonatal } \\
\text { Intensive Care Unit Network }\end{array}$ & $\begin{array}{l}\text { LPI had lower adjusted scores } \\
\text { for attention }(p=.04) \text {, arousal } \\
(p=.01) \text {, regulation }(p<.001)\end{array}$ & 15 \\
\hline
\end{tabular}


Table 1. Continued

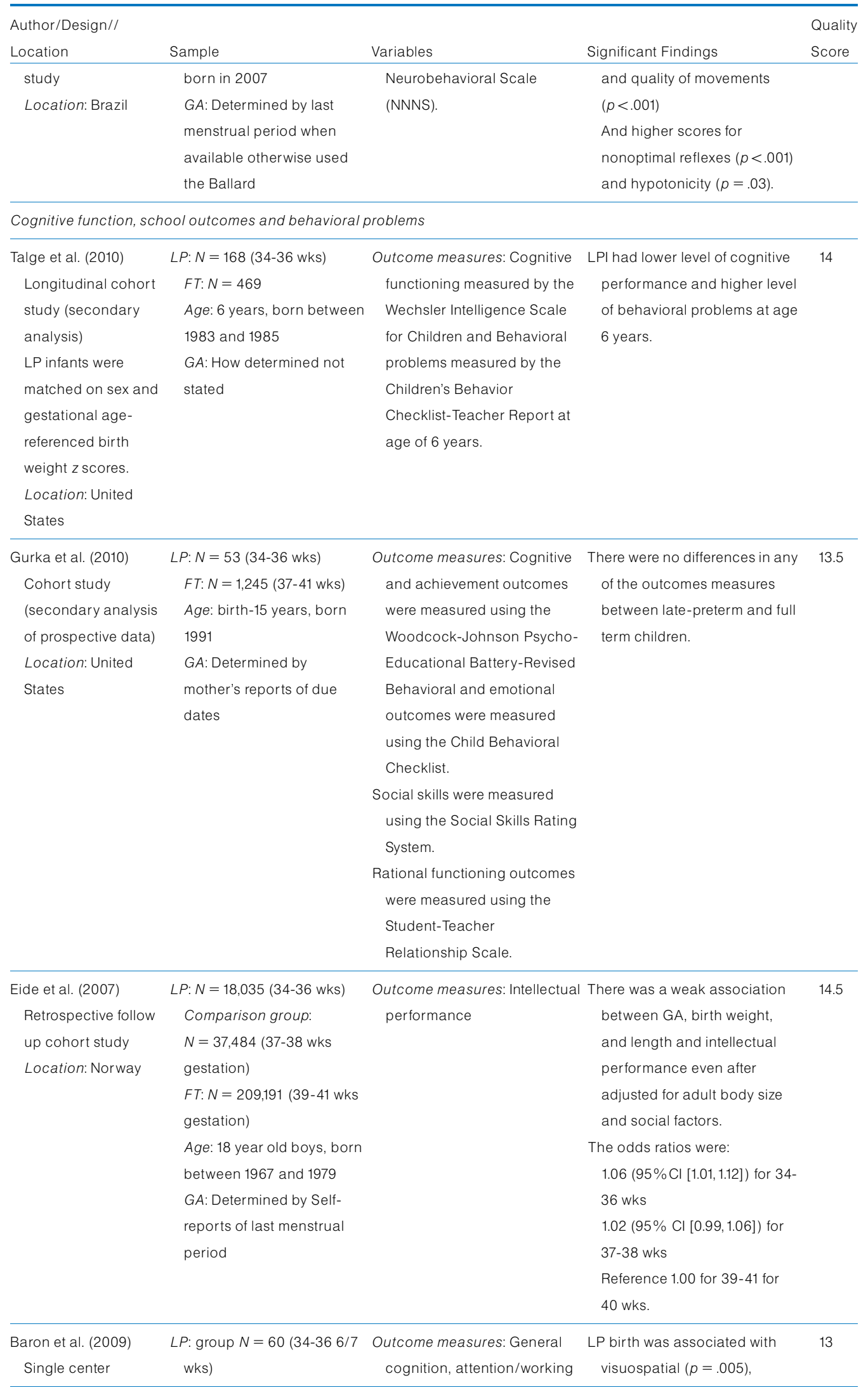




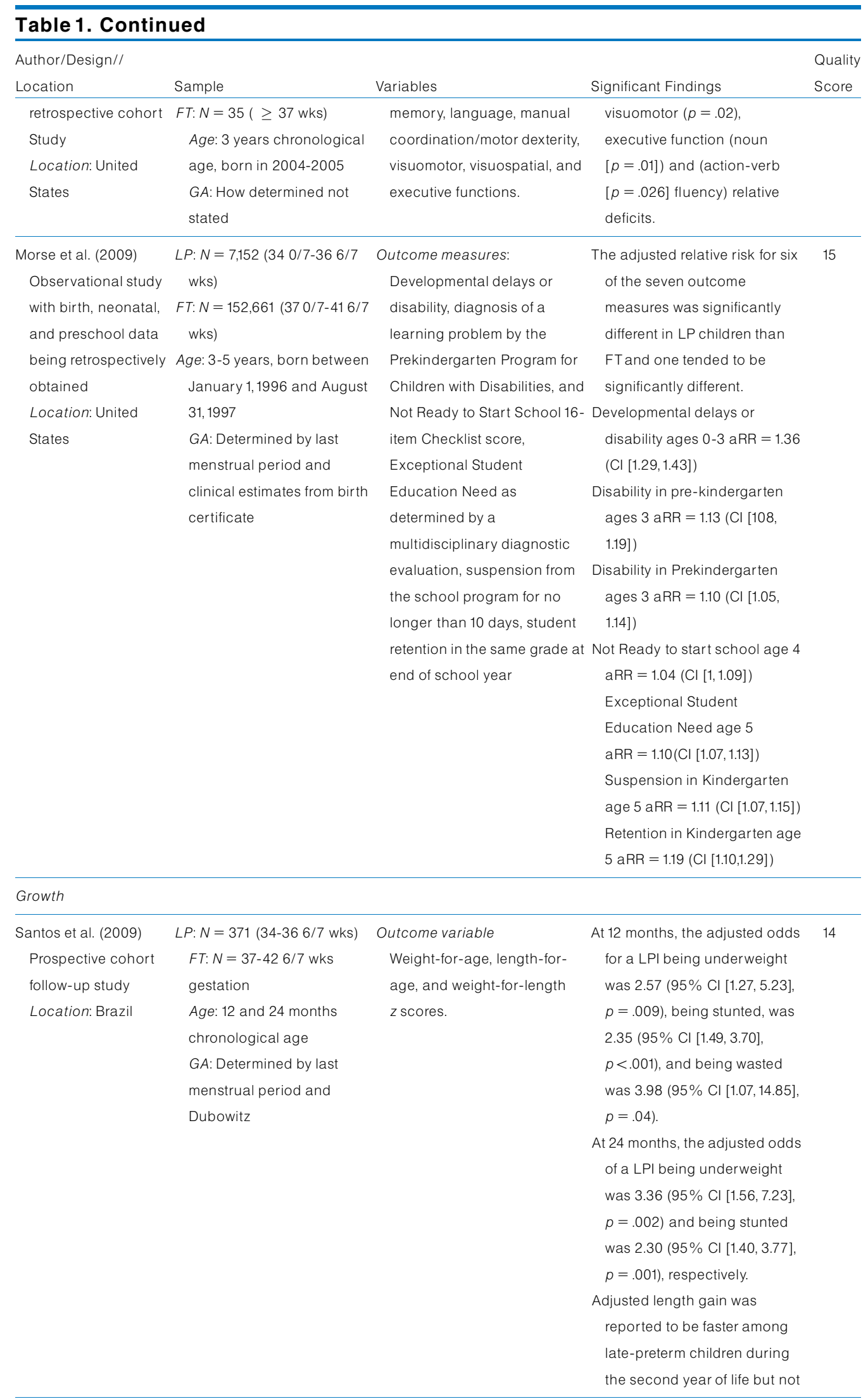




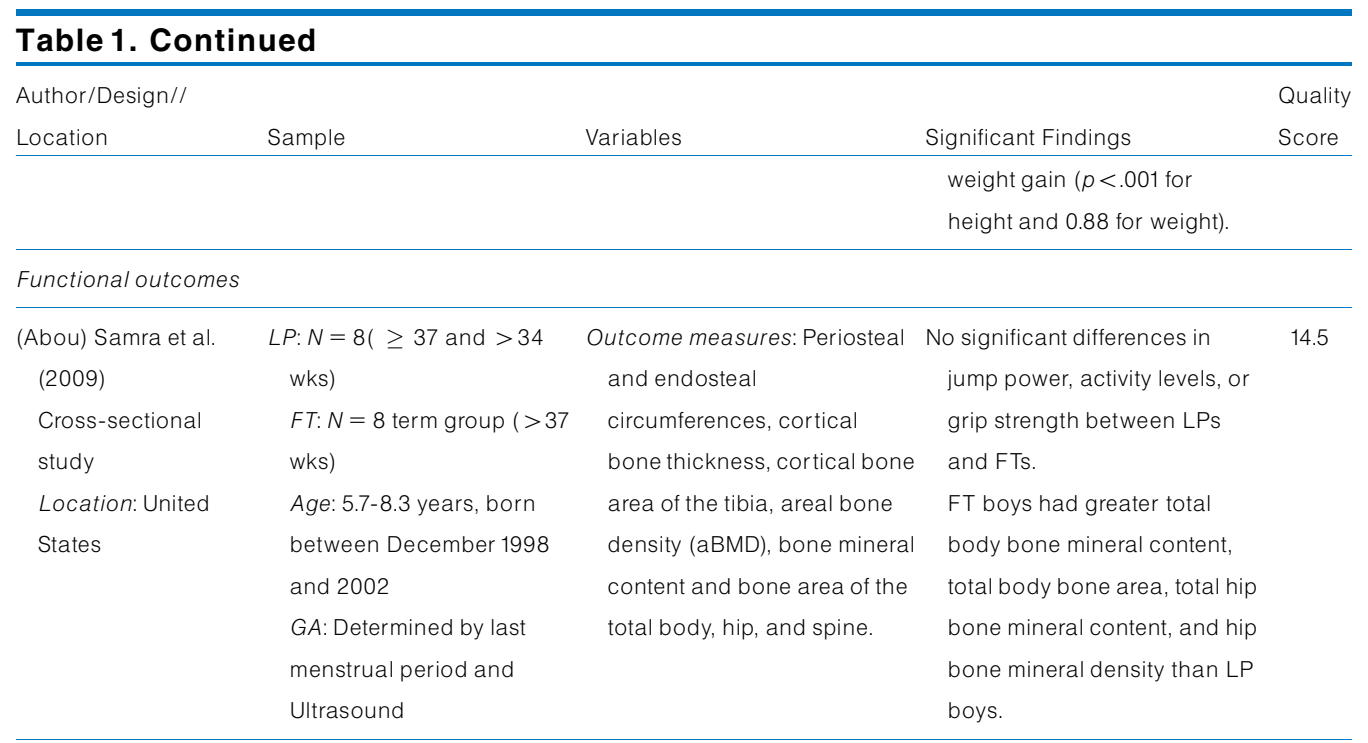

Note. $\mathrm{CA}=$ corrected age; $\mathrm{FT}(\mathrm{I})=$ full term (infants); GA = gestational age; $\mathrm{LP}(\mathrm{I})=$ late-preterm (infant); wks = weeks; aRR = adjusted relative risk; $\mathrm{Cl}=$ confidence intervals.

and cerebral palsy were grouped under neurodevelopmental outcomes in Domain I. Studies that examined attention, arousal, and regulation and psychosocial outcomes with the exception of studies that examined attention deficit disorder were grouped under neuro-behavioral outcomes in Domain II. Because studies on behavioral and psychosocial performance with special focus on attention deficit disorder, withdrawal and anxiety, aggressive behavior, depression, and somatic complaints examined those outcomes in relation to school achievement, cognitive outcomes, need for special education, and intelligence quotient (IQ), those studies were included under cognitive and school outcomes in Domain III. Studies addressing age-appropriate growth using weight and height and head circumference and measurement of body composition such as percentage body fat, lean mass, skin fold thickness, and biochemical analysis to assess for adequate nutrition were grouped under Domain IV. Studies examining the ability of the child to perform age appropriate daily tasks and activities as well as physical performance including dressing, bathing, feeding, mobility, communication, social interaction, and sports participation were grouped under functional outcomes in Domain V. Table 1 summarizes the studies first in relationship to their outcome domain and then in chronological order. Reports of statistically significant findings were included in the table when comparisons between $\mathrm{LPI}$ and $\mathrm{FTI}$ were made.
These included odds ratios, relative risks, confidence intervals, and $p$ values.

In the following section, findings of the review studies are discussed following the same sequence as in Table 1, except for the two analyses by Romeo et al. (2007, 2009). Even though the analyses were performed at two different time periods, both addressed neurodevelopmental outcomes and appeared to come from the same cohort. Therefore, the 2007 analysis has been discussed in conjunction with the 2009 analysis and does not follow the chronological order.

Neuro-Developmental Outcomes. Four of the studies addressed issues related to neurodevelopmental outcomes (Domain I). A retrospective study by Petrini et al. (2009) was designed to assess the association between LP birth and incidence of adverse neurodevelopmental outcomes including cerebral palsy, developmental delays/mental retardation, and presence of seizure disorders. Latepreterm infants were reported to have higher risk for cerebral palsy, mental retardation, and developmental delays than their 5- to 6-year-old FT counterparts. Participants of the study were recruited from Kaiser Permanente Medical Care follow-up comprehensive health care coverage program. Study limitations included its retrospective design and the risk for incorrect or missed diagnoses as the ICD-9-CM codes were the only 


\section{Table 2: Modified Downs and Black Quality Scoring System for Evaluating Observational Studies on Long-Term Outcomes in Late-Preterm (Infant)}

\begin{tabular}{l} 
Quality Indicators \\
\hline Reporting
\end{tabular}

\begin{tabular}{ll}
\hline External validity & \\
\hline Are study participants representative of entire & 1 \\
population form which they were recruited & \\
\hline
\end{tabular}

If described somewhere else give a point

Are study participants representative of current late- 1 preterm population

Were those participants who were prepared to $\quad 1$

participate representative of entire population form

which they were recruited

Internal validity

Was an attempt made to blind those measuring main $\quad 1$ outcomes of the study

Did the study adjust for different lengths of follow-up 1

of participants

\begin{tabular}{ll}
\hline Were statistical tests used appropriate & 1 \\
\hline
\end{tabular}

Was the outcome measure used accurate $\quad 1$

Were the participants in the different groups recruited 1

from the same populations

Were study participants in the different groups recruited 1

over the same time period

Was there adequate adjustment of confounding in 1 the analysis

Study design

Cross-sectional 0.5

Cohort study prospective (2 points)

Control group (yes $=1$ point, no $=0$ points $)$

\section{Table 2. Continued}

\begin{tabular}{lc}
\hline Quality Indicators & Points \\
\hline Secondary/Retrospective analysis (1 point) & 1 \\
\hline Sample size adequate/power analysis (yes =1 for, & 1 \\
no $=0$ ) & \\
\hline Total max points for study design (4 points) & 4 \\
\hline Total points & 20 \\
\hline
\end{tabular}

method used to identify the presence of disease states. In addition, a significant percentage of study participants were of ethnic minorities. Even though the study adjusted for ethnicity, psychosocial disparities could have been a factor that modified the effect of gestation on the outcome. The authors did not report on the severity of the diagnosed disabilities, or on whether the presence of any neonatal complications such as hypoxia, acidosis, sepsis, etc. could have influenced their results. Such complications are believed to contribute to the development of periventricular leukomalacia (PVL), which even though its incidence in LPI is for the most part unknown, it is reported to be the most common reason for neurological disabilities in preterm infants (Kinney, 2006; Resch et al., 2006).

In two separate secondary analyses completed with what seems to be two separate samples drawn from the same cohort, Romeo et al. $(2007,2009)$ assessed neurological development in LPIs using the Hammersmith Infant Neurological Examination (HINE) in one sample (Romeo et al., 2007) and the Forward Parachute Reaction (FPR) and age of independent walking in the other (Romeo et al., 2009). In the first analysis, LPIs scored lower than the normative published scores for FTI for tone and reflexes. In the second analysis, FTs and LPIs who had complete FPR development by 9 months of age attained independent walking at a median age of 13 months. Corrected gestational age was used for LPIs. Both studies did not adjust for neonatal complications that could have confounded the results. Both studies did not include a FT comparison group, and comparisons were limited to published data. Being retrospective secondary analyses, both studies are limited in their generalizability.

With the aim of examining the relationship between gestational age at birth and long-term medical and social outcomes, Moster et al. (2008) conducted a follow-up study on a sample of 903,402 young adults who were born in Norway between 1967 and 1983. The follow-up period 
extended from those infants born from 1967 to 2003 and thus, the ages of the participants at the time of the study ranged from 20 to 36 years. Moster et al. found that as gestational age group (23-27 6/7 weeks gestations [Wks], 28-30 6/7 Wks, 31-33 6/7 Wks, 34-36 6/7 Wks, and $\geq 37$ Wks) increases, the risk for medical disabilities including cerebral palsy and mental retardation decreases. Those findings are consistent with the previous analysis by Petrini et al. (2009). Furthermore, Moster et al. reported that risk for social outcomes such as unemployment, inability to establish a family, and lower education attainment increases as gestational age category becomes lower. However, Moster et al. did not report on how risk for medical disabilities, cerebral palsy, mental retardation, rate of unemployment, inability to establish a family, and lower education attainment in the LP group compared with that of the FT group. The first cohort of study participants was born 44 years ago. Since then neonatal care has improved considerably, particularly with improved respiratory support, special attention to pain management and developmental interventions, and thus the span of time for those participants included in the study could be a significant limitation. Given those improvements in the standard of neonatal care, it remains uncertain whether the same results would be observed among the current late-preterm population if followed into the future. In addition, neonatal morbidities as well as other socioeconomic factors that the study did not account for could have confounded study findings.

Psychosocial and Neurobehavioral Behavioral Outcomes. The search yielded two studies that examined psychosocial and behavioral outcomes in LP infants (Domain II). However, one of the studies examined the behavioral outcomes in relation to school and cognitive performance and therefore will be included in the cognitive domain. The other study by Barros et al. (2010) was designed to compare neuro-behavior in LPIs with that of FTIs born to adolescent mothers during the first 24 to 72 hours of life. Late-preterm infants were shown to have lower scores for attention, arousal, regulation, and quality of movements and higher scores for nonoptimal reflexes and hypertonicity than infants born at term on the Neonatal Intensive Care Unit Network Neurobehavioral Scale (NNNS) after adjusting for several covariates including type of anesthesia that the mother received prior to delivery, mode of delivery, age of the infant at the time of examination, and time of feedings. Lack of a control group of nonadolescent mothers with FTIs and LPIs is a limitation of the study as well as the fact that there is no follow-up data to corroborate those results.
Cognitive and School Outcomes. The search yielded five studies that were included in Domain III. In a 6-year follow-up longitudinal study on infants born between 1983 and 1985, Talge et al. (2010) found that LPIs had lower level of cognitive performance and higher level of behavioral problems at age 6 years when compared with their FT counterparts who were matched on sex and gestational age-referenced birth weight $z$ scores. Although Gurka et al. (2010) found no differences in cognitive performance and rational functioning skills between LP and FT adolescents born in 1991, Eide et al. (2007) reported lower intellectual performance in adolescents with history of late-preterm birth and who were born between 1967 and 1979. Morse et al. (2009) reported higher risk for learning disabilities and need for special education or learning assistance in school-age LP children born between 1996 and 1997. Among 3-year-old children who were recruited from one of the tertiary neonatal intensive care units (NICUs) in the United States, Baron et al. (2009) found significant association between late-preterm birth and deficits in visuomotor and executive functions.

Both studies by Talge et al. (2010) and by Eide et al. (2007) were secondary analyses with study participants born 28 to 44 years ago. The generalizability of those findings, given recent advances in neonatal care and in obstetric practices, is limited; and it would be difficult to compare findings from those cohorts with findings from current LP populations. In addition, the study by Eide et al. was limited to boys and gestational age classification was limited to maternal reports.

The study by Gurka et al. (2010) was a secondary analysis and had a small sample size of LP participants $(N=53)$. Participants were recruited from 10 different sites and tended to be of higher socioeconomic status and educational level. These demographic data could have significantly confounded study findings. In the Morse et al.'s study (2009), mothers of late-preterm infants were more likely to be African American, younger, of lower educational and socioeconomic status, and to have had experienced a higher rate of complications during pregnancy, labor, and deliver. Again, these demographic data may have confounded the study findings. Baron's sample was limited to infants admitted to one tertiary-level NICU. No information was provided on their level of care on their neonatal morbidities.

Although findings from the above studies raises concerns about increased risk for suboptimal school performance and cognitive functioning in 
LPIs, there are several limitations to existing data, and a conclusive statement about school and cognitive outcomes in LPIs cannot be reached. Cognitive and school performance is influenced by several factors. Impairments of executive function, visual and motor skills, and memory lead to learning disabilities. Ecological, policy, and psychosocial factors including geographic locations, cultural and ethnic background, and individual motivation also have a great influence on school and cognitive performance. Assessing cognitive and school performance is complex and subject to several confounding factors such as reliance on subjective and inconsistent measures in assessing school performance, poor definitions of maternal and neonatal health conditions and comorbidities, and lack of reliable and consistent methodology for gestational age estimates. Last, some of the tests used to measure cognitive performance are age specific and provide little insight into long-term outcomes.

Growth. Our search yielded one study that was included in this domain (VI). In a prospective cohort study designed to investigate the influence of LP birth on growth, Santos et al. (2009) examined weight-for-age, length-for-age, and weight-forlength z-scores in a sample of 371 LPIs at 12 and 18 months of age and compared to those of FTIs. At 12 months of age, LPIs were found to be underweight, stunted, and wasted compared to full-term infants. Even though, weight gain was similar in both groups during the second year of life, length gain was found to be accelerated in LPIs. The investigators did not use corrected gestational age when comparing outcome variables. It is not clear whether correction for gestational age would have significantly influenced those differences and whether weight differences were due to overall difference in muscle mass or lean mass.

In a cross-sectional study designed to determine whether there are differences in bone mass and size between LP and FT prepubertal boys and whether those differences are explained by differences in activity levels, (Abou) Samra et al. (2009) found no differences in body mass index (BMI), lean body mass, of percentage body fat between LP and FT boys aged 5.7 to 8.3 years. Those results suggest that LPI catch up to their FT counterparts sometime during early infancy or childhood. It is unclear as to whether suboptimal growth observed during the first 2 years of life in other populations (Santos et al., 2009) is reversed later in early childhood. The age of catch up in growth in LPIs needs further exploration. Age-appropriate weight gain and growth is an indicator of adequate nutrition. In addition, gaining insights on various aspects and patterns of growth in early in infancy and childhood could be of great importance to understanding risks for developing chronic diseases such as obesity, heart disease, osteoporosis, and diabetes later in life, and if those risks are greater for LPIs.

Activity and Functional Outcomes. Our search yielded one study that was included in Domain V. The cross-sectional study by (Abou) Samra et al. (2009), as previously described found no differences in jump height and power, grip strength, or physical activity levels between LP and FT boys. However, the study found that LP boys had lower bone mass and smaller bone size than FT boys at several bone sites after adjusting for several covariates including age, body weight, and height. Although bone mass and size to a great extent are genetically determined, nutrition and activity in early childhood are believed to have a significant impact on bone accretion. Due to the small sample size, and due to the use of parental reports to measure physical activity, lack of differences in physical performance between LP and FT children cannot be confirmed. Additional research is needed to examine functional performance in LP children and the mechanisms through which it may have an impact of development and growth including bone accretion.

\section{Practice Recommendation and Research Implications}

\section{Practice Recommendations}

The development of brain structural and functional processes that takes place during the last few weeks of gestation and continues into the early postnatal period is modulated by several biopsychosocial and environmental factors. A mature brain may regulate and adapt differently than an immature brain when challenged with various external and internal stimuli. Early experiences can have lifelong effects on growth and development. Preventing adverse experiences and tailoring interventions to meet the unique needs that are special to late-preterm infants is the best strategy to influence long-term outcomes. Immediate caregivers play an important role in structuring and providing such environment and in employing practices that promote optimal growth and development and minimizes the risks for adverse outcomes. Infants who exhibit poor neurobehavioral performance in the early perinatal period are at risk for poor long-term outcomes (Liu et al., 2010). Providing care with regard to the infant's neurobehavioral states, minimizing bright and loud stimuli, and promoting 
maternal bonding are examples of such preventive strategies. Strategies such as effective management of apnea and bradycardia and preventing fluctuation in cerebral blood flow can also have a protective effect against cerebral injury which is believed to be a precursor for PVL (Verhagen, Keating, ter Horst, Martijn, \& Bos, 2009). However, because this is a group that has not routinely been screened or followed for PVL it is difficult to know where these same strategies are applicable to this population. More prospective trials with well-designed interventions are needed to provide direction in this area to practitioners.

Even though current evidence does not appear to be conclusive at this time and data are very limited, the general consensus is that LPI are at increased risk for suboptimal long-term outcomes. Risk of adverse outcomes may not be evident at the time of hospital discharge and may be compounded by several modifiable factors, thus necessitating timely assessment and follow-up care. Assessment and surveillance beyond the early neonatal period are critical in identifying individuals at risk. Providing education on potential risks can be of utmost importance as a means to best support optimal outcomes for these infants. Low socioeconomic status, lack of family support, and limited education may increase the biological burdens of late-preterm birth.

\section{Research Implication}

A small number of studies exists that examined growth and developmental outcomes in LPIs. Even though current evidence regarding suboptimal growth and developmental outcomes in LPIs is limited and does not appear to be conclusive, the general consensus is that risk for suboptimal outcomes increases as gestation at birth decreases.

Significant variations in the literature exist regarding study methodology and populations. Standardization of future study protocols and latepreterm classification and definition are needed, so that risk for suboptimal outcomes can be appropriately appraised. This review revealed that only 12 studies (3 cross-sectional studies, 2 prospective studies, and 7 retrospective analyses) met the LP definition of 34 to 36 6/7 weeks gestation which was recommended by the 2005 workshop "Optimizing Care and Outcome of the Near-Term Pregnancy and the Near-Term Newborn Infant" sponsored by the National Institutes of Health (Engle et al., 2008). Participants in four of the studies were born more than 20 years ago. In a fifth study participants were born 13 years ago. Three of the analyses did not include a comparison group, and two were cross-

\section{Preventing adverse experiences and tailoring interventions to meet the unique needs of late-preterm infants is the best strategy to influence outcomes.}

sectional studies. Longitudinal prospective studies with full-term comparison groups are needed to better characterize risks associated with LP birth and to identify pathways to disparities in outcomes, so that future research can focus on identifying the most effective intervention and reducing the burdens of biological risk associated with LP birth. Several questions remain unanswered and longitudinal studies to determine whether outcomes observed at a specific age continues into the future. Among those questions are the following:

- Is the risk for suboptimal growth and development limited to infants with neonatal complications or does the risk extend to those who had an uneventful neonatal transition?

- Are some of the observed delays and problems in LPIs a lag in development, or are these delays associated with permanent disabilities?

- Are delays global or limited to specific areas of development, and what are those areas?

- What psychosocial, maternal, and neonatal characteristics contribute to increased risk, and how do these factors modify the risk?

- Are there windows of opportunity during which interventions will lead to the best possible outcome?

\section{Limitations}

This integrative review was aimed at summarizing and evaluating existing evidence supporting suboptimal growth and developmental outcomes in late-preterm infants born between 34 and 36 6/7 weeks gestation. Due to limited resources and time constraints, author contacts were not sought, and the search was limited to the English language. Differences in participant characteristics, late preterm classification, inclusion and exclusion of multiple gestations, methods of gestational age estimation, outcomes of interest, and methodology exist. Lack of random selection, risk of confounding, description of the population as it currently exists, risk of loss to follow-up, and the retrospective design of the examined studies that are general limitations to cohort studies are also limitations to this review.

\section{Conclusion}

Available data on long-term developmental outcomes in the late-preterm population come 


\section{Longitudinal prospective studies with full-term comparison groups are needed to better appraise risks associated with late-preterm birth and to identify pathways to decrease disparities.}

predominantly from retrospectively studies with the majority of the participants born prior the last decade or even as much as 40 years ago. Since then neonatal care has improved considerably, particularly with special attention to respiratory care, pain management, and developmental interventions. Therefore, the generalizability of the findings is limited, and it remains uncertain whether the same results would be observed among the current latepreterm population if followed into the future. Differences in obstetric practices and management of neonatal morbidities from one population to another as well as differences in socioeconomic and demographic characteristics of the study participants also limit generalizability. It is unclear whether risks for suboptimal outcomes in LPI are limited to specific areas of development or are more global in nature and whether risks are limited to certain vulnerable subgroups, or extend to those who had uneventful neonatal and prenatal histories. Due to heterogeneity and paucity of data, there is no clear characterization of long-term risks associated with late-preterm birth. The developmental trajectory of an infant is determined by a complex interplay among the infant's biological vulnerabilities, several environmental variables, and psychosocial factors. Strategies aimed at minimizing risks and optimizing growth and development can only be successful when those risks and contributing factors are well understood. This is an area where much work is still needed.

\section{REFERENCES}

(Abou) Samra, H., Binkley, T., Stevens, D., \& Specker, B. (2009). Determinants of bone mass and size in 7-year-old former term, late-preterm, and preterm boys. Osteoporosis International, 20, 192-201, doi:10.1007/s00198-009-0896-z.

Baron, I. S., Erickson, K., Ahronovich, M. D., \& Coulehan, K. (2009). Visuospatial and verbal fluency relative deficits in "complicated" late-preterm preschool children. Early Human Development, 85, 751-754.

Barros, M. C. M., Mitsuhiro, S., Chalem, E., \& Laranjeira, R. R. (2010). Neurobehavior of late-preterm infants of adolescent mothers. Neonatology, 99, 133-139.

Bastek, J. A., Sammel, M. D., Pare, E., Srinivas, S. K., Posencheg, M. A., \& Elovitz, M. A. (2008). Adverse neonatal outcomes: Examining the risks between preterm, late preterm, and term infants. American Journal of Obstetrics and Gynecology, 199, 367.e1-367.e8, Retrieved from http://www.ncbi.nlm.nih.gov/pubmed/18928976.

Downs, S. H., \& Black, N. (1998). The feasibility of creating a checklist for the assessment of the methodological quality both of randomised and non-randomised studies of healthcare interventions. Journal of Epidemiology and Community Health, 52, 377-384.

Eide, M. G., Oyen, N., Skjaerven, R., \& Bjerkedal, T. (2007). Associations of birth size, gestational age, and adult size with intellectual performance: Evidence from a cohort of Norwegian men. Pediatric Research, 62, 636-642.

Engle,W.,Tomashek, K. M., \& Wallman, C.American Academy of Pediatrics Committee on Fetus and Newborn. (2008). ACOG committee opinion: Late-preterm infants. Obstetrics and Gynecology, 11, 1029-1031.

Gurka, M. J., LoCasale-Crouch, J., \& Blackman, J. A. (2010). Long-term cognition, achievement, socioemotional, and behavioral development of healthy late-preterm infants. Archives of Pediatric and Adolescent Medicine, 16, 525-532.

Kinney, H. C. (2006). The near-term (late-preterm) human brain and risk for perventicular leukomalacia: A review. Seminars in Perinatology, 30, 81-88.

Liu, J., Bann, C., Lester, B., Tronick, E., Das, A., \& Lagasse, L. (2010). Neonatal neurobehavior predicts medical and behavioral outcome. Pediatrics, 125, e90-e98, Retrieved from http://www.ncbi.nlm.nih. gov/pmc/articles/PMC2873896/. doi:10.1542/peds.2009-0204.

Martin, A. J., Osterman, M. J. K., \& Sulton, P. D. (2010). Are preterm births on the decline in the United States? Recent data from the National Vital Statistics System ( $(\mathrm{NCHS}$ data National Center For Health Statistics Brief, No. 39) Hyattsville, MD: National Vital Statistics System.

Melnyk, B. M., Feinstein, N., \& Fairbanks, E. (2006). Two decades of evidence to support implementation of the COPE program as standard practice with parents of young unexpectedly hospitalized/critically ill children and premature infants. Pediatric Nursing, 32, $475-481$.

Morse, S. B., Zheng, H., Tang, Y., \& Roth, J. (2009). Early school-age outcomes of late preterm infants. Pediatrics, 123, e623-e629, Retrieved from http://www. pediatrics.org/cgi/content/full/123/4/e622.

Moster, D., Terje Lie, R., \& Markestad, T. (2008). Long-term medical and social consequences of preterm birth. New England Journal of Medicine, 359, 262-273.

Petrini, J. R., Dias, T., McCormick, M. C., Massolo, M. L., Green, N., \& Escobar, J. (2009). Increased risk of adverse neurological development for late preterm infants. Journal of Pediatrics, 154, 169-176.

Resch, B., Jammernegg, A., Perl, E., Riccabona, M., Maurer, U., \& Muller, W. D. (2006). Correlation of grading and duration of periventricular echodensities with neurodevelopmental outcomes in preterm children. Pediatric Radiology, 36, 810-815.

Romeo, D. M., Cione, A. L., Guzzetta, A., Scoto, M., Conversano, M., Palermo, F., \& Merculi, E. (2007). Application of a scorable neurological examination to near-term infants: Longitudinal data. Neuropediatrics, 38, 233-238.

Romeo, D. M., Cioni, M., Scoto, M., Palermo, F., Pizzardi, A., \& Sorge, A. (2009). Development of the forward parachute reaction and the age of walking in near term infants: A longitudinal observational study. BioMed Central Pediatrics, 9, Retrieved from http://www. bio medcentral.com/147-2431/9/13. doi:10:1186/1471-2431/9/13.

Santos, I. S., Matijasevich, A., Domingues, M. R., Barros, A. J. D., Victora, C. G., \& Barros, F. C. (2009). Late preterm birth is a risk factor for growth faltering in early childhood: A cohort study. BioMed Central Pediatrics, 9, Retrieved from http://www.biomedcentral.com/ 1471-2431/9/71. doi:10.1186/1471-2431-9-71.

Stroup, F. D., Berlin, J. A., Morton, S. C., Olkin, I., Rennie, D., \& Moher, D. (2000). Meta-analyses of observational studies in epidemiology. Journal of the American Medical Association, 283, 2008-2012.

Talge, N. M., Holzman, C., Wang, J., Lucia, V., Gardiner, J., \& Breslau, N. (2010). Late-preterm birth and its association with cognitive and socioemotional outcomes at 6 years of age. Pediatrics, 126, 11241131. 
Tomashek, K., Shapiro-Mendoza, C. K., Davidoff, M., \& Petrini, J. R. (2007). Differences in mortality between late-preterm and term singleton infants in the United States, 1995-2002. Journal of Paediatrics, 151, 450-456.

Tonse, N. K., Higgins, R. D., Stark, A., \& Leveno, K. J. (2006). Optimizing care and outcome of late-preterm (near-term) infants: A summary of the workshop sponsored by National Institute of Child Health and Human Development. Pediatrics, 118, 1207-1218.

Verhagen, E. A., Keating, P., ter Horst, H. J., Martijn, A., \& Bos, A. F. (2009). Cerebral saturation and extraction in preterm infants with transient periventricular echodensities. Pediatrics, 124, 294-301.
Wang, M. L., Dorer, D. J., Fleming, M. P., \& Catlin, E. A. (2004). Clinical outcomes of near-term infants. Pediatrics, 114, 372-376

West, S., King, V., Carey, T. S., Lohr, K. N., McKoy, N., \& Sutton, S. F. (2002). Systems to rate the strength of scientific evidence: Evidence report/technology assessment number 47 ((AHRQ Publication No. 02-E016) Rockville, MD: Agency for Healthcare Research and Quality. Retrieved from http://www.thecre.com/pdf/ahrq-sys tem-strength.pdf.

White-Traut, R., \& Norr, K. (2009). An ecological model for premature infant feeding. Journal of Obstetric, Gynecology, \& Neonatal Nursing, 38, 478-490, doi:10.1111/j.1552-6909.01046.x. 